\title{
Discrimination between Malignant and Benign Vertebral Fractures Using Magnetic Resonance Imaging
}

\author{
Tomoyuki Takigawa ${ }^{1}$, Masato Tanaka ${ }^{1}$, Yoshihisa Sugimoto ${ }^{1}$, \\ Tomoko Tetsunaga ${ }^{1}$, Keiichiro Nishida ${ }^{1,2}$, Toshifumi Ozaki ${ }^{1}$ \\ ${ }^{1}$ Department of Orthopaedic Surgery, Okayama University Hospital, Okayama, Japan \\ ${ }^{2}$ Department of Human Morphology, Okayama University Graduate School of Medicine, Density and Pharmaceutical Sciences, Okayama, Japan
}

Study Design: Retrospective analysis using magnetic resonance imaging (MRI).

Purpose: To identify MRI features that could discriminate benign from malignant vertebral fractures.

Overview of Literature: Discrimination between benign and malignant vertebral fractures remains challenging, particularly in patients with osteoporosis and cancer. Presently, the most sensitive means of detecting and assessing fracture etiology is MRI. However, published reports have focused on only one or a few discriminators.

Methods: Totally, 106 patients were assessed by MRI within six weeks of sustaining 114 thoracic and/or lumbar vertebral fractures (benign, $\mathrm{n}=65$; malignant, $\mathrm{n}=49$ ). The fractures were pathologically confirmed if malignant or clinically diagnosed if benign and were followed up for a minimum of six months. Seventeen features were analyzed in all fractures' magnetic resonance images. Single parameters were analyzed using the chi-square test; a logit model was established using multivariate logistic regression analysis.

Results: The chi-square test revealed 11 malignant and 4 benign parameters. Multivariate logistic regression analysis selected (i) posterior wall diffuse protrusion (odds ratio [OR], 48; 95\% confidence interval [CI], 4.2-548; $p=0.002$ ), (ii) pedicle involvement (OR, $21 ; 95 \% \mathrm{Cl}, 2.0-229 ; p=0.01$ ), (iii) posterior involvement (OR, 21; $95 \% \mathrm{Cl}, 1.5-21 ; p=0.02$ ), and (iv) band pattern (0R, 0.047; $95 \% \mathrm{Cl}$, $0.0005-4.7 ; p=0.19$ ). The logit model was expressed as $\mathrm{P}=1 /[1+\exp (\mathrm{x})], \mathrm{x}=-3.88 \times(\mathrm{i})-3.05 \times(\mathrm{ii})-3.02 \times(\mathrm{iii})+3.05 \times(\mathrm{iv})+5.00$, where $P$ is the probability of malignancy. The total predictive value was $97.3 \%$. The only exception was multiple myeloma with features of a benign fracture.

Conclusions: Although each MRI feature had a different meaning with a variable differentiation power, combining them led to an accurate diagnosis. This study identified the most relevant MRI features that would be helpful in discriminating benign from malignant vertebral fractures.

Keywords: Spine; Spinal fracture; Osteoporosis; Metastasis; Logistic model

\section{Introduction}

Osteoporotic vertebral compression fractures are common among older people. The clinical prognosis is generally benign because these fractures usually heal without complications. Spinal metastasis or primary neoplasms can

Received Oct 14, 2016; Revised Nov 17, 2016; Accepted Nov 20, 2016

Corresponding author: Tomoyuki Takigawa

Department of Orthopaedic Surgery, Okayama University Hospital,

2-5-1 Shikata-cho, Kita-ku, Okayama City, Okayama 700-8558, Japan

Tel: +81-86-235-7273, Fax: +81-86-223-9727, E-mail: takigawa2004@yahoo.co.jp 
also cause pathological vertebral fractures in this particular patient population. However, their clinical course is usually malignant because of the risk of progressive spinal destruction that causes persistent pain and neurological deficits. Spinal fractures must be accurately diagnosed because the clinical course, prognosis, and treatment strategies for malignant and benign fractures are very different. Magnetic resonance imaging (MRI) is reportedly the most sensitive modality to detect and assess the etiology of spinal fractures [1-3]. However, many published reports have focused on only one or a few discriminators. The present study aimed to identify MRI features that could discriminate benign from malignant vertebral fractures and to establish a predictive model to discriminate the etiology of spinal fractures.

\section{Materials and Methods}

We assessed 106 patients (mean age, 71.5 years) using MRI within six weeks of sustaining 114 thoracic and/or lumbar vertebral fractures (benign, $n=65$; malignant, $n=49$ ). These fractures were either pathologically confirmed or clinically diagnosed and were followed up for a minimum of six months at our institute between 2004 and 2014. Malignant fractures were pathologically confirmed, and most benign fractures were clinically diagnosed as decrudescence, although disappearance of symptoms and bony union were found within a minimum follow-up of six months. Malignant fractures included metastases of lung $(n=9)$, prostate $(n=7)$, breast $(n=5)$, thyroid $(n=4)$, kidney $(n=4)$, and gastric cancers and other malignant pathologies comprising hepatocarcinoma, ureteral cancer, uterine cancer, osteosarcoma, malignant lymphoma, multiple myeloma, bile duct carcinoma, leiomyosarcoma, gingival cancer, alveolar soft-part sarcoma, and adenocarcinoma of unknown origin. A surgeon with over 10 years of experience in treating spinal fractures analyzed 17 known MRI features described below in all fractures. Band pattern [46]; fluid signs [5,7]; complete replacement [8]; endplate involvement [9]; compression of the entire body [4]; low and high signal intensity of lesions on T1- and T2-weighted images, respectively [4]; and disc involvement [10] were analyzed in the vertebral body. Posterior wall diffuse protrusion $[4,11,12]$, posterior focal protrusion $[4,11,12]$, and an absent black line indicating the posterior vertebral margin on T2 [8] were analyzed in the posterior wall. Posterior $[4,11,12]$ and pedicle $[4,8,13]$ involvement were analyzed in the posterior column and pedicle, respectively. The MRI features of epidural $[4,12,13]$ and paraspinal $[4,13,14]$ masses, other bone marrow lesions $[4,15]$, and other fractures with $[9,13]$ and without $[4,10]$ changes in bone marrow signals were also analyzed. Our institutional Ethics Review Board approved the study protocol.

Single parameters were analyzed using chi-square tests. A logit model was established using multivariate logistic regression analysis with sequential elimination at $p>0.2$. Statistical significance was set at $p<0.05$. All data were statistically analyzed using Excel Statistics 2012 (SSRI Co. Ltd., Tokyo, Japan).

\section{Results}

The chi-square test revealed 11 malignant and 4 benign parameters (Table 1). The malignant features comprised pedicle involvement, posterior wall diffuse protrusion, epidural mass, paraspinal mass, posterior involvement, disappearance of the T2 black line on posterior wall, presence of other bone marrow lesions, and complete compression with low signal intensity on T1-weighted images. The benign features comprised band pattern, posterior wall focal protrusion, fluid signs, and the presence of other previous benign fractures. All these features were statistically significant with variable sensitivity (23\%-96\%) and specificity (58\%-98\%). Table 2 shows that multivariate logistic regression analysis selected the malignant features of posterior wall diffuse protrusion (odds ratio [OR], 48; 95\% confidence interval [CI], 4.2-548; $p=0.002$ ), pedicle involvement (OR, 21; 95\% CI, 2.0-229; $p=0.01$ ), and posterior involvement (OR, 21; 95\% CI, 1.5-21; $p=0.02)$, and the sole benign feature of band pattern (OR, 0.047; 95\% CI, $0.0005-4.7 ; p=0.19$. The logit model was expressed as follows:

$$
\mathrm{P}=1 /[1+\exp (\mathrm{x})], \mathrm{x}=-3.88 \times(\mathrm{i})-3.05 \times(\mathrm{ii})-3.02 \times(\mathrm{iii})+3.0
$$
$5 \times($ iv) +5.00 ,

where $\mathrm{P}$ is the probability of malignancy, (i) is posterior wall diffuse protrusion, (ii) is pedicle involvement, (iii) is posterior involvement, and (iv) is band pattern.

Positive and negative features were assigned values of 1 and 0 , respectively. Although the $p$-value of band pattern did not reach $<0.05$, this variable was included in the multivariate analysis because the $p$-value was $<0.2$. The total calculated predictive value using this logit model was 97.3\%. The only exception in the present series was pathologically confirmed multiple myeloma. 
Table 1. Results of univariate analysis of $17 \mathrm{MRI}$ features

\begin{tabular}{|c|c|c|c|c|c|}
\hline MRI feature & Univariate OR & $95 \% \mathrm{Cl}$ & $p$-value & Sensitivity (\%) & Specificity (\%) \\
\hline Pedicle involvement & 270 & $50-1,456$ & $<0.001$ & 95.7 & 92.3 \\
\hline Posterior wall diffuse protrusion & 224 & $48-1,050$ & $<0.001$ & 93.6 & 93.8 \\
\hline Epidural mass & 160 & $32-792$ & $<0.001$ & 95.7 & 87.7 \\
\hline Paraspinal mass & 94 & $12-720$ & $<0.001$ & 59.6 & 98.5 \\
\hline Posterior involvement & 76 & $20-296$ & $<0.001$ & 78.7 & 95.4 \\
\hline Disappearance of black line on T2 & 36 & $4.6-285$ & $<0.001$ & 36.2 & 98.5 \\
\hline Other bone marrow lesion & 19 & $6.5-57$ & $<0.001$ & 61.7 & 92.3 \\
\hline Complete replacement & 12 & $4.1-34$ & $<0.001$ & 89.4 & 58.5 \\
\hline Compression of all body & 9.8 & $3.8-25$ & $<0.001$ & 85.1 & 63.1 \\
\hline Other fractures with bone marrow changes & 3.1 & $1.3-7.7$ & $<0.01$ & 36.2 & 84.6 \\
\hline T1 low and T2 high lesion & 2.5 & $1.2-5.5$ & $<0.05$ & 51.1 & 70.8 \\
\hline End plate involvement & 1.3 & $0.59-2.7$ & $>0.05$ & 59.6 & 46.2 \\
\hline Disc involvement & 1.0 & $0.42-2.5$ & $>0.05$ & 23.4 & 76.9 \\
\hline Other fractures without signal changes & 0.16 & $0.055-0.45$ & $<0.001$ & 43.1 & 89.4 \\
\hline Fluid sign & 0.15 & $0.032-0.68$ & $<0.001$ & 23.1 & 95.7 \\
\hline Posterior wall focal protrusion & 0.053 & $0.0068-0.41$ & $<0.001$ & 29.2 & 97.9 \\
\hline Band pattern & 0.049 & $0.0063-0.38$ & $<0.001$ & 30.8 & 97.9 \\
\hline
\end{tabular}

Odds ratios of $>1$ and $<1$ indicate higher probability for malignant or benign fracture, respectively.

$\mathrm{MRI}$, magnetic resonance imaging; $\mathrm{OR}$, odds ratio; $\mathrm{Cl}$, confidence interval.

Table 2. Results of multivariate logistic regression analysis

\begin{tabular}{lccc} 
MRI feature & Multivariate OR & $95 \% \mathrm{Cl}$ & $p$-value \\
\hline Posterior wall diffuse protrusion & 48 & $4.3-548$ & 0.0017 \\
\hline Pedicle involvement & 21 & $2.0-229$ & 0.012 \\
\hline Posterior involvement & 21 & $1.5-283$ & 0.024 \\
\hline Band pattern & 0.047 & $0.0005-4.7$ & 0.19 \\
\hline
\end{tabular}

$\mathrm{MRI}$, magnetic resonance imaging; $\mathrm{OR}$, odds ratio; $\mathrm{Cl}$, confidence interval.

\section{Discussion}

Our univariate analysis results showed that each MRI feature had a different meaning and variable differentiation power and that these features were more common and more significant when the fracture was malignant. These results corresponded to those of a systemic review by Thawait et al. [16] who associated other non-characteristic vertebral lesions, paraspinal masses, involvement of the posterior element, involvement of the pedicle, complete replacement of normal bone marrow, epidural mass, and diffuse convexity of the posterior vertebral border with malignant fractures, whereas coexisting healed benign vertebral fractures, fluid signs, focal posterior vertebral border retropulsion, and a band-like abnormal signal were associated with benign fractures. Our multivariate analysis of the current MRI findings selected the malignant features of posterior wall diffuse protrusion, pedicle and posterior involvement, and the benign features of a band pattern. Combining these four MRI features allowed accurate diagnoses with a predictive value of $97.3 \%$. Yuzawa et al. [8] accurately diagnosed 99 of 100 spinal fractures using a simple scoring system based on the benign findings of a preserved normal bone marrow signal, a continuous black line on T2 and a clear fracture line, the malignant findings of pedicle or other posterior element involvement, expansion into the paravertebral region, and osteolytic destruction; These authors, however, analyzed 
Table 3. Examples of provability of malignancy according to four MRI features

\begin{tabular}{lcccccccc}
\hline Posterior wall diffuse protrusion & + & + & + & - & + & - & - & - \\
Pedicle involvement & + & + & - & + & - & + & - & - \\
Posterior involvement & + & - & + & + & - & - & + & - \\
Band pattern & - & - & - & - & - & - & - & - \\
Probability of malignancy (\%) & 99.3 & 87.4 & 87.1 & 74.7 & 24.8 & 12.6 & 12.3 & 0.68 \\
\hline
\end{tabular}

$\mathrm{MRI}$, magnetic resonance imaging; +, positive; -, negative.

computed tomography (CT) as well as MRI data. Differences in methodology and patient data might explain these conflicting results. Diffusion-weighted imaging (DWI) is reportedly useful for differentiating acute osteoporotic compression fractures from malignant vertebral fractures [16-19]. The apparent diffusion coefficient is lower for malignant fractures than for benign fractures. Adding DWI to conventional MRI improves diagnostic accuracy from $92 \%$ to $98 \%$ [19]. Nevertheless, the clinical application of DWI remains limited because some technical factors, such as the definition of regions of interest and MRI protocols, might influence the results $[16,19]$.

Probability was easily calculated using our logit model (Table 3). The probability of malignancy was $99.3 \%$ when the three features of posterior wall diffuse protrusion, pedicle involvement, and posterior involvement were evident and a band pattern was absent and was $75 \%-87 \%$ when two of the three malignant parameters were positive without a band pattern. Figs 1 and 2 show typical malignant and benign fractures, respectively. When two or more malignant features are positive but the primary

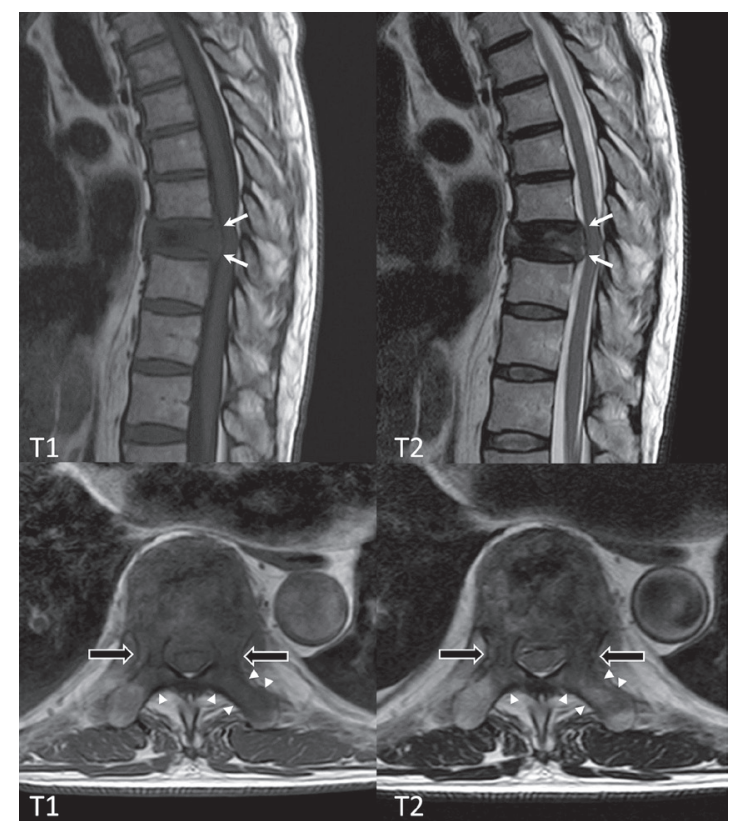

Fig. 1. Malignant vertebral fracture due to metastasis of lung cancer. Magnetic resonance image shows posterior wall diffuse protrusion (white arrows), pedicle involvement (black arrows), and posterior involvement (arrowheads) at T9.

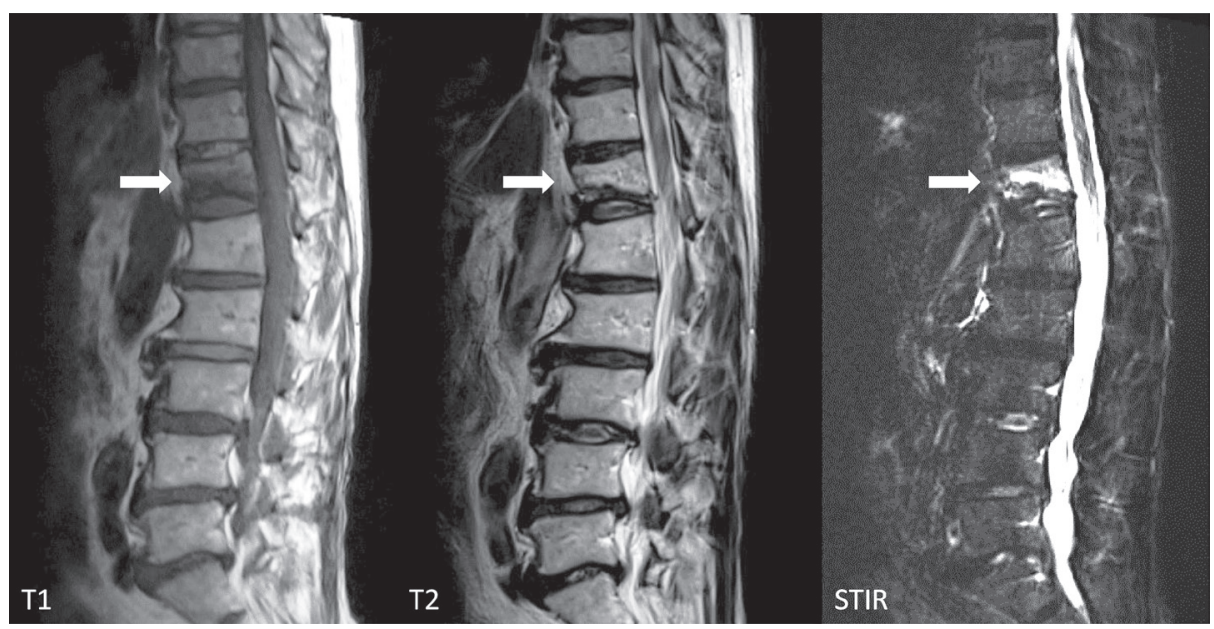

Fig. 2. Benign vertebral fracture due to osteoporosis. Magnetic resonance image shows a band pattern at T12 that appears as areas of low and high signal intensity on T1-weighted and short-tau inversion recovery (STIR) images, respectively (white arrows). 
lesion is unknown, further examinations such as wholebody CT assessment, tumor marker examination, positron emission tomography-computed tomography, and biopsy are required because the probability of a pathological fracture is high. Even when only one malignant feature is positive, patients should be further examined in this manner because the probability of malignancy is $>12 \%$. On the other hand, the probabilities of malignancy were $0.03 \%$ and $0.68 \%$ when all three malignant features were negative with and without a band pattern, respectively. Benign (osteoporotic) fractures are more likely under these circumstances, and invasive biopsy should be avoided even if a patient has a history of cancer.

We had only one exception. Although MRI findings indicated a benign fracture due to the absence of posterior wall diffuse protrusion, pedicle or posterior involvement, and a fluid sign, the fracture was associated with pathologically confirmed multiple myeloma. This result corresponded with the findings of a previous study in which the features of $67 \%$ and $33 \%$ of 224 spinal fractures in patients with multiple myeloma were benign and malignant, respectively [20]. Patients with fractures and multiple myeloma should be very carefully assessed because a low body mass index, low levels of albumin, and increased levels of myeloma protein, light chains, and creatinine are risk factors for spinal fracture [21].

\section{Conclusions}

We identified the most relevant MRI features that could facilitate the discrimination of benign from malignant vertebral fractures.

\section{Conflict of Interest}

No potential conflict of interest relevant to this article was reported.

\section{References}

1. Tan DY, Tsou IY, Chee TS. Differentiation of malignant vertebral collapse from osteoporotic and other benign causes using magnetic resonance imaging. Ann Acad Med Singapore 2002;31:8-14.

2. An HS, Andreshak TG, Nguyen C, Williams A, Daniels D. Can we distinguish between benign versus malignant compression fractures of the spine by magnetic resonance imaging? Spine (Phila Pa 1976) 1995;20:1776-82.

3. Cicala D, Briganti F, Casale L, et al. Atraumatic vertebral compression fractures: differential diagnosis between benign osteoporotic and malignant fractures by MRI. Musculoskelet Surg 2013;97 Suppl 2:S169-79.

4. Jung HS, Jee WH, McCauley TR, Ha KY, Choi KH. Discrimination of metastatic from acute osteoporotic compression spinal fractures with MR imaging. Radiographics 2003;23:179-87.

5. Baur A, Stabler A, Arbogast S, Duerr HR, Bartl R, Reiser M. Acute osteoporotic and neoplastic vertebral compression fractures: fluid sign at MR imaging. Radiology 2002;225:730-5.

6. Moulopoulos LA, Yoshimitsu K, Johnston DA, Leeds NE, Libshitz HI. MR prediction of benign and malignant vertebral compression fractures. J Magn Reson Imaging 1996;6:667-74.

7. Ragab Y, Emad Y, Gheita T, et al. Differentiation of osteoporotic and neoplastic vertebral fractures by chemical shift \{in-phase and out-of phase\} MR imaging. Eur J Radiol 2009;72:125-33.

8. Yuzawa Y, Ebara S, Kamimura M, et al. Magnetic resonance and computed tomography-based scoring system for the differential diagnosis of vertebral fractures caused by osteoporosis and malignant tumors. J Orthop Sci 2005;10:345-52.

9. Rupp RE, Ebraheim NA, Coombs RJ. Magnetic resonance imaging differentiation of compression spine fractures or vertebral lesions caused by osteoporosis or tumor. Spine (Phila Pa 1976) 1995;20:2499-503.

10. Yuh WT, Zachar CK, Barloon TJ, Sato Y, Sickels WJ, Hawes DR. Vertebral compression fractures: distinction between benign and malignant causes with MR imaging. Radiology 1989;172:215-8.

11. Lafforgue P, Bayle O, Massonnat J, et al. MRI in osteoporotic and metastatic vertebral compressions: apropos of 60 cases. Ann Radiol (Paris) 1991;34:15766.

12. Cuenod CA, Laredo JD, Chevret S, et al. Acute vertebral collapse due to osteoporosis or malignancy: appearance on unenhanced and gadolinium-enhanced MR images. Radiology 1996;199:541-9.

13. Fu TS, Chen LH, Liao JC, Lai PL, Niu CC, Chen WJ. Magnetic resonance imaging characteristics of benign and malignant vertebral fractures. Chang Gung Med J 2004;27:808-15. 
14. Shih TT, Huang KM, Li YW. Solitary vertebral collapse: distinction between benign and malignant causes using MR patterns. J Magn Reson Imaging 1999;9:635-42.

15. Abanoz R, Hakyemez B, Parlak M. Diffusion-weighted imaging of acute vertebral compression: Differential diagnosis of benign versus malignant pathologic fractures. Tani Girisim Radyol 2003;9:176-83.

16. Thawait SK, Marcus MA, Morrison WB, Klufas RA, Eng J, Carrino JA. Research synthesis: what is the diagnostic performance of magnetic resonance imaging to discriminate benign from malignant vertebral compression fractures? Systematic review and metaanalysis. Spine (Phila Pa 1976) 2012;37:E736-44.

17. Balliu E, Vilanova JC, Pelaez I, et al. Diagnostic value of apparent diffusion coefficients to differentiate benign from malignant vertebral bone marrow lesions. Eur J Radiol 2009;69:560-6.
18. Pozzi G, Garcia Parra C, Stradiotti P, Tien TV, Luzzati A, Zerbi A. Diffusion-weighted MR imaging in differentiation between osteoporotic and neoplastic vertebral fractures. Eur Spine J 2012;21 Suppl 1:S1237.

19. Sung JK, Jee WH, Jung JY, et al. Differentiation of acute osteoporotic and malignant compression fractures of the spine: use of additive qualitative and quantitative axial diffusion-weighted MR imaging to conventional MR imaging at 3.0 T. Radiology 2014; 271:488-98.

20. Lecouvet FE, Vande Berg BC, Maldague BE, et al. Vertebral compression fractures in multiple myeloma. Part I. Distribution and appearance at MR imaging. Radiology 1997;204:195-9.

21. Miller JA, Bowen A, Morisada MV, et al. Radiologic and clinical characteristics of vertebral fractures in multiple myeloma. Spine J 2015;15:2149-56. 